\title{
Desfechos Hospitalares em Pacientes Submetidos a Intervenção Coronária Percutânea na Vigência de Síndromes Coronárias Agudas Atendidos em Unidades de Pronto Atendimento (UPAs) - Resultados de um Centro de Cardiologia Terciário
}

\author{
Fernando Mendes Sant'Anna', Fernando Suarez Alvarez², Ricardo Vasconcellos Bruno ${ }^{2}$, \\ Marcelo Bastos Brito ${ }^{1}$, Sérgio Menezes', Wilson Braz Correa Filho², \\ Carlos Alberto Mussel Barrozo ${ }^{1}$, Sérgio Luiz Côrtes da Silveira ${ }^{2}$
}

\section{RESUMO}

Introdução: A criação das Unidades de Pronto Atendimento (UPAs) veio atender a uma necessidade urgente do governo brasileiro em melhorar o atendimento à população carente. Rapidamente as UPAs se tornaram centros de referência para tratamento da dor torácica nessa população, em decorrência da agilidade do atendimento, da disponibilidade de medicamentos de última geração e da existência de pessoal treinado. Este estudo visa a descrever o perfil e a evolução hospitalar de pacientes com síndrome coronária aguda atendidos nessas unidades e posteriormente encaminhados a um hospital terciário, onde foram tratados por angioplastia com implante de stent. Método: No período de julho a dezembro de 2009, 300 pacientes foram encaminhados das UPAs para o Hospital Santa Helena (Cabo Frio, RJ). Desses pacientes, 164 (202 lesões) foram submetidos a implante de stent e divididos em dois grupos: grupo I, formado por 86 pacientes com síndrome coronária aguda com supradesnível do segmento ST; e grupo II, formado por 78 pacientes com síndrome coronária aguda sem supradesnível do segmento ST. Os dois grupos foram comparados em relação a suas características clínicas e angiográficas e a eventos cardíacos adversos maiores hospitalares. Resultados: As características clínicas e angiográficas foram similares nos dois grupos, com exceção do tabagismo. Foi obtido sucesso angiográfico em $99 \%$ dos procedimentos. O tempo médio de internação (UPA + hospital) foi de seis dias. Houve 6 eventos cardíacos adversos maiores hospitalares, 5 dos quais no grupo I e 1 no grupo II (5,8\% vs. $1,3 \%$; $\mathrm{P}=0,60)$. Conclusão: $O$ tratamento percutâneo tardio dos pacientes

\author{
ABSTRACT \\ In-Hospital Outcomes in Patients with Acute \\ Coronary Syndromes Undergoing Coronary \\ Percutaneous Intervention Primarily Assisted in \\ the Emergency Care Units (ECUs) - Results \\ from a Tertiary Cardiology Center
}

Background: Emergency Care Units (ECUs) were created to meet an urgent need of the Brazilian government to improve health care to low-income population. ECUs quickly became reference centers to treat chest pain in this population, due to their ability to provide prompt medical care, availability of last generation drugs and well trained staff. This study is aimed at describing the profile and inhospital follow-up of patients with acute coronary syndromes seen in these units and later referred to a tertiary hospital, where they were treated by percutaneous coronary intervention with stent implantation. Method: Three hundred patients were referred from ECUs to Santa Helena Hospital (Cabo Frio, RJ) from July to December, 2009. One hundred and sixty four patients (202 lesions) were treated by percutaneous intervention with stent and were divided in two groups: group I included 86 patients with acute coronary syndromes with ST segment elevation and group II included 78 patients with acute coronary syndromes without ST segment elevation. Clinical and angiographic characteristics and in-hospital major adverse cardiac events were compared between the two groups. Results: Clinical and angiographic characteristics were similar in both groups except for smoking. Angiographic success was obtained in $99 \%$ of the procedures. Mean hospitalization time (ECU +

\footnotetext{
Hospital Santa Helena - Cabo Frio, RJ, Brasil.

2 Secretaria de Estado de Saúde e Defesa Civil do Estado do Rio de Janeiro - Rio de Janeiro, RJ, Brasil.

Correspondência: Fernando Mendes Sant'Anna. Rua Safira, 20 -

Portinho - Cabo Frio, RJ, Brasil - CEP 28915-400

E-mail: fernandomendes@cardiol.br

Recebido em: 7/1/2010 • Aceito em: 9/3/2010
} 
Sant'Anna FM, et al. Desfechos Hospitalares em Pacientes Submetidos a Intervenção Coronária Percutânea na Vigência de Síndromes Coronárias Agudas Atendidos em Unidades de Pronto Atendimento (UPAs) - Resultados de um Centro de Cardiologia Terciário. Rev Bras Cardiol Invasiva. 2010;18(1):30-6.

com síndrome coronária aguda atendidos nas UPAs é um procedimento seguro e eficaz, cursando com altas taxas de sucesso e baixos índices de complicações hospitalares.

DESCRITORES: Stents. Infarto do miocárdio. Revascularização miocárdica.

A s doenças cardiovasculares, principalmente o infarto agudo do miocárdio, permanecem sendo maior causa de óbito e incapacidade no Brasil e no mundo. ${ }^{1,2} \mathrm{O}$ estudo Global Registry of Acute Coronary Events (GRACE), um dos maiores registros de síndromes coronárias agudas e que tem o Brasil como um dos países participantes, mostra índice de óbito hospitalar de $11 \%$ nesse grupo de pacientes. ${ }^{3}$

A partir de 2000, o infarto agudo do miocárdio contou com nova definição, que incluía outros marcadores de necrose miocárdica, como as troponinas I ou T e a mioglobina. ${ }^{4}$ Com isso, entre $10 \%$ e $38 \%$ de pacientes com angina instável foram posteriormente reclassificados como infarto agudo do miocárdio, aumentando em 25\% a incidência dessa condição clínica. Esses pacientes, caracteristicamente, evoluem com mortalidade 1,5 a 3 vezes maior que aqueles com enzimas normais, no curto prazo. ${ }^{4}$ Dessa maneira, por apresentarem risco mais elevado, merecem cuidados médicos e tratamentos especializados, tanto na fase aguda como fase na tardia.

Apesar da melhora crescente no atendimento aos pacientes carentes pelo Sistema Único de Saúde (SUS), ainda existe dificuldade no acesso às tecnologias de ponta e a hospitais terciários. O sistema hospitalar de nosso País encontra-se sobrecarregado em algumas cidades, como o Rio de Janeiro, e raramente os pacientes têm a chance de ser atendidos em tempo hábil e posteriormente fazer acompanhamento adequado, visando à prevenção de novos eventos.

De maneira a melhorar o atendimento do paciente SUS, foram criadas as Unidades de Pronto Atendimento (UPAs), cujo objetivo é atender rapidamente o paciente que a elas recorre, diagnosticando, tratando e transferindo os pacientes que necessitem internação para hospitais terciários da rede pública ou conveniados ao SUS. Essas unidades são administradas pelo Corpo de Bombeiros do Estado do Rio de Janeiro, subordinado à Secretaria de Estado de Saúde e Defesa Civil do Rio de Janeiro (SESDEC/RJ). Os pacientes com dor torácica aguda são levados a essas unidades, onde fazem eletrocardiograma e têm marcadores de necrose miocárdica dosados. Quando se diagnostica uma síndrome coronária aguda, com ou sem supradesnível do segmento ST, o paciente é tratado segundo as tertiary hospital) was 6 days. There were 6 in-hospital major adverse cardiac events, 5 in group I and 1 in group II $(5.8 \%$ vs. $1.3 \%$; $\mathrm{P}=0.60)$. Conclusion: Late percutaneous revascularization of patients with acute coronary syndromes seen at ECUs is a safe and effective procedure, with high success and low complication rates.

KEY-WORDS: Stents. Myocardial infarction. Myocardial revascularization.

Diretrizes da Sociedade Brasileira de Cardiologia ${ }^{5,6}$ e, depois, encaminhado para um hospital.

O objetivo deste estudo foi analisar o índice de complicações hospitalares nesse grupo de pacientes encaminhados ao Hospital Santa Helena (Cabo Frio, $\mathrm{RJ}$ ), comparando as características clínicas e angiográficas e os eventos hospitalares de pacientes com síndromes coronárias agudas com e sem supradesnível do segmento ST.

\section{MÉTODO}

Entre julho e dezembro de 2009, 300 pacientes foram encaminhados das UPAs (22 no total, todas localizadas na cidade do Rio de Janeiro) para o Hospital Santa Helena. Todos apresentavam diagnóstico de síndrome coronária aguda com ou sem supradesnível do segmento ST. Desses pacientes, 262 (87,3\%) apresentavam doença aterosclerótica coronária e 164 $(54,7 \%)$ foram submetidos a angioplastia com implante de stent, constituindo a população deste estudo. Esses pacientes eram trazidos diretamente de suas UPAs de origem ou de uma UPA central, de ambulância, para Cabo Frio. A distância média percorrida é de $160 \mathrm{~km}$ e o tempo de transporte gira em torno de duas horas e meia.

Os pacientes foram trazidos para o Hospital Santa Helena em média 4 dias após terem sido internados nas UPAs, onde foram tratados clinicamente. A angioplastia com implante de stent foi realizada em todos os casos em que se identificava vaso relacionado ao evento agudo a ser tratado. Não foram realizados testes indutores de isquemia nesse grupo de pacientes previamente às intervenções. Pacientes que apresentassem doença grave no tronco da coronária esquerda (> 50\%) foram encaminhados para tratamento cirúrgico, salvo se existisse alguma contraindicação.

Os pacientes, para efeito de análise, foram divididos em dois grupos: grupo I, pacientes com síndrome coronária aguda com supradesnível do segmento ST (86 casos); e grupo II, pacientes com síndrome coronária aguda sem supradesnível do segmento ST (78 casos). As características clínicas, angiográficas e os eventos hospitalares foram comparados nesses grupos. 
Sant'Anna FM, et al. Desfechos Hospitalares em Pacientes Submetidos a Intervenção Coronária Percutânea na Vigência de Síndromes Coronárias Agudas Atendidos em Unidades de Pronto Atendimento (UPAs) - Resultados de um Centro de Cardiologia Terciário. Rev Bras Cardiol Invasiva. 2010;18(1):30-6.

O objetivo primário do estudo foi avaliar as taxas de eventos cardíacos adversos maiores hospitalares nesses pacientes. Definiu-se eventos cardíacos adversos maiores como a incidência combinada de óbito por qualquer causa, (re)infarto agudo do miocárdio, acidente vascular cerebral ou nova revascularização da lesão-alvo. O infarto agudo do miocárdio foi definido como aumento da CK-MB > 3 vezes o limite superior do normal ou aumento de 50\% da CK-MB basal, se esta estivesse aumentada.

\section{Intervenção coronária percutânea}

O implante dos stents nos pacientes do estudo foi realizado por via femoral, utilizando-se cateteres guia $6 \mathrm{~F}$ ou $7 \mathrm{~F}$. Antes da angioplastia, eram administradas $10.000 \mathrm{UI}$ de heparina endovenosa e 0,5 mg de nitroglicerina intracoronária. Todos os pacientes faziam uso prévio de ácido acetilsalicílico e ticlopidina ou clopidogrel, iniciados três dias antes do procedimento. Caso o paciente não fizesse esse preparo, era administrada dose de ataque de $300 \mathrm{mg}$ de clopidogrel e $100 \mathrm{mg}$ de ácido acetilsalicílico antes do procedimento. Após o procedimento, os pacientes eram orientados a manter o ácido acetilsalicílico indefinidamente e o tienopiridínico por 30 dias.

Sempre que possível foi realizado implante direto do stent. Os stents eram escolhidos de modo a cobrir inteiramente a lesão e mais de um stent era permitido em caso de dissecção de borda, cobertura incompleta da lesão ou resultado subótimo. Os stents utilizados eram não-farmacológicos: PRO Kinetic (Biotronik Inc., Lake Oswego, Estados Unidos), BX Sonic ou Presillion (Cordis Inc., Miami, Estados Unidos), Tsunami (Terumo, Tóquio, Japão), e Liberté (Boston Scientific Corp., Marlborough, Estados Unidos).

O sucesso do procedimento foi definido como obtenção de lesão residual $<30 \%$ e ausência de complicações maiores (morte ou infarto) nas primeiras 24 horas após o procedimento.

\section{Angiografia coronária quantitativa}

A angiografia coronária quantitativa foi realizada off-line, utilizando-se software com algoritmo de detecção automática de contorno (CAAS II, Pie Medical Imaging, Maastricht, Holanda), conforme descrito previamente. ${ }^{7}$

\section{Análise estatística}

Todas as variáveis foram testadas para normalidade pelo teste de Shapiro-Wilks. Na análise descritiva, as variáveis numéricas foram apresentadas como média \pm desvio padrão ou medianas e interquartis e as categóricas, como números (n) e porcentagens (\%). O teste de qui-quadrado e o teste exato de Fisher foram usados para variáveis categóricas; o teste $t$ de Student não-pareado e o teste de Mann-Whitney foram usados para variáveis contínuas. As características clínicas e angiográficas foram comparadas entre os grupos síndrome coronária aguda com supradesnível do seg- mento ST e síndrome coronária aguda sem supradesnível do segmento ST. A análise estatística foi realizada com o programa $R$ Statistic 2.10. Valores de $\mathrm{P}<0,05$ (bicaudal) foram considerados estatisticamente significantes.

\section{RESULTADOS}

Durante um período de seis meses consecutivos, 300 pacientes foram admitidos no Hospital Santa Helena provenientes das UPAs. Destes, 164 (54,7\%) foram submetidos a angioplastia com implante de stent, sendo 86 pertencentes ao grupo síndrome coronária aguda com supradesnível do segmento ST e 78 ao grupo síndrome coronária aguda sem supradesnível do segmento ST. A mediana do tempo de internação desses pacientes foi de 7 (intervalo interquartil, 5-8) dias, sendo 4 (intervalo interquartil, 3-6) deles nas UPAs de origem e 2 (intervalo interquartil, 2-2) no Hospital Santa Helena.

Os pacientes portadores de síndrome coronária aguda com supradesnível do segmento ST receberam trombolíticos em 69\% (59/86) dos casos. Desses, 90\% apresentavam a artéria culpada aberta no momento do cateterismo, comparativamente a $63 \%$ dos pacientes que não receberam trombolíticos. Dentre os trombolíticos, em $85 \%$ dos casos foi utilizada a tenecteplase em bolus e em 15\%, a alteplase. Os pacientes que não receberam trombolíticos foram tratados clinicamente de acordo com protocolo de dor torácica utilizado nas UPAs. Todos os pacientes encaminhados se encontravam estáveis após o tratamento inicial do infarto agudo do miocárdio. Não foi realizada nenhuma angioplastia de resgate pós-trombólise.

As características clínicas desses pacientes podem ser analisadas na Tabela 1. Chama a atenção o alto índice de diabéticos na população estudada (33,5\%), principalmente no grupo síndrome coronária aguda com supradesnível do segmento ST (41\% vs. 26\% no grupo síndrome coronária aguda sem supradesnível do segmento $\mathrm{ST} ; \mathrm{P}=0,05)$. A única característica clínica que se mostrou estatisticamente diferente entre os dois grupos foi o tabagismo $(32,3 \%$ do total), que predominou no grupo síndrome coronária aguda com supradesnível do segmento ST (42\% vs. $22 \% ; \mathrm{P}=0,007)$.

As características angiográficas das lesões podem ser observadas na Tabela 2. A maior parte das lesões era do tipo B2/C (55\%). Não houve diferença entre os diâmetros de referência ou em relação ao comprimento da lesão entre os dois grupos e o grau médio de estenose pela angiografia coronária quantitativa foi de $76 \%$. Nota-se também predominância de artéria descendente anterior tratada no grupo síndrome coronária aguda com supradesnível do segmento ST em relação ao grupo síndrome coronária aguda sem supradesnível do segmento ST $(54,4 \%$ vs. $38,4 \%)$. Uma minoria $(12,4 \%)$ das lesões abordadas apresentava fluxo TIMI 0 ou 1 no momento da intervenção. Esses pacientes foram trata- 
Sant'Anna FM, et al. Desfechos Hospitalares em Pacientes Submetidos a Intervenção Coronária Percutânea na Vigência de Síndromes Coronárias Agudas Atendidos em Unidades de Pronto Atendimento (UPAs) - Resultados de um Centro de Cardiologia Terciário. Rev Bras Cardiol Invasiva. 2010;18(1):30-6.

TABELA 1

Características clínicas dos pacientes

\begin{tabular}{|c|c|c|c|c|}
\hline & \multirow[b]{2}{*}{$\begin{array}{l}\text { Pacientes } \\
(n=164)\end{array}$} & \multicolumn{2}{|c|}{ Grupos } & \multirow[b]{2}{*}{ Valor de $\mathbf{P}$} \\
\hline & & $\begin{array}{c}\text { SCACSST } \\
(n=86)\end{array}$ & $\begin{array}{c}\text { SCASSST } \\
(n=78)\end{array}$ & \\
\hline Idade & $60,8 \pm 11,1$ & $60,9 \pm 10,8$ & $60,8 \pm 11,6$ & 0,95 \\
\hline Sexo feminino, n (\%) & $63(38)$ & $29(33,7)$ & $34(43,6)$ & 0,20 \\
\hline \multicolumn{5}{|l|}{ Fatores de risco, n (\%) } \\
\hline Hipertensão arterial & $126(76,8)$ & $61(71)$ & $65(83)$ & 0,07 \\
\hline Dislipidemia & $77(47)$ & $38(44)$ & $39(50)$ & 0,53 \\
\hline HF de DAC & $69(42)$ & $36(42)$ & $33(42)$ & $>0,99$ \\
\hline Diabetes & $55(33,5)$ & $35(41)$ & $20(26)$ & 0,05 \\
\hline Tabagismo & $53(32,3)$ & $36(42)$ & $17(22)$ & 0,007 \\
\hline IAM prévio, n (\%) & $31(19)$ & $12(14)$ & $19(24)$ & 0,11 \\
\hline ICP prévia, n (\%) & $2(1,2)$ & $1(1,2)$ & $1(1,3)$ & $>0,99$ \\
\hline RM prévia, n (\%) & $3(1,8)$ & $1(1,2)$ & $2(2,6)$ & 0,60 \\
\hline Fração de ejeção, \% & $53 \pm 11$ & $52 \pm 11$ & $55 \pm 12$ & 0,24 \\
\hline Doença multiarterial, n (\%) & $109(66,5)$ & $55(64)$ & $54(69)$ & 0,51 \\
\hline Stent por paciente & $1,32 \pm 0,60$ & $1,27 \pm 0,56$ & $1,37 \pm 0,85$ & 0,45 \\
\hline
\end{tabular}

TABELA 2

Características angiográficas - análise por lesão

\begin{tabular}{|c|c|c|c|c|}
\hline & \multirow[b]{2}{*}{$\begin{array}{c}\text { Lesões } \\
(n=202)\end{array}$} & \multicolumn{2}{|c|}{ Grupos } & \multirow[b]{2}{*}{ Valor de P } \\
\hline & & $\begin{array}{l}\text { SCACSST } \\
(n=103)\end{array}$ & $\begin{array}{c}\text { SCASSST } \\
(\mathrm{n}=99)\end{array}$ & \\
\hline Artéria coronária, n (\%) & & & & 0,04 \\
\hline Descendente anterior & $94(46,5)$ & $56(54,4)$ & $38(38,4)$ & \\
\hline Coronária direita & $52(25,7)$ & $26(25,2)$ & $26(26,3)$ & \\
\hline Circunflexa & $27(13,4)$ & $8(7,8)$ & $19(19,2)$ & \\
\hline Outros vasos & $29(14,4)$ & $13(12,6)$ & $16(16,2)$ & \\
\hline Lesão B2/C (ACC/AHA), n (\%) & $112(55,4)$ & $53(51,5)$ & $59(59,6)$ & 0,48 \\
\hline DR pré-ICP, mm & $2,92 \pm 0,53$ & $2,93 \pm 0,51$ & $2,91 \pm 0,55$ & 0,81 \\
\hline DLM pré-ICP, mm & $0,69(0,47 ; 0,96)$ & $0,72(0,53 ; 0,98)$ & $0,65(0,45 ; 0,94)$ & 0,22 \\
\hline DS pré-ICP, \% & $76(68 ; 83)$ & $76(67 ; 82)$ & $76(69 ; 84)$ & 0,27 \\
\hline Comprimento da lesão, mm & $14,6(10,4 ; 20,7)$ & $14,2(11,2 ; 19,1)$ & $14,6(10 ; 22,7)$ & 0,43 \\
\hline DR pós-ICP, mm & $3,20 \pm 0,53$ & $3,20 \pm 0,48$ & $3,20 \pm 0,57$ & 0,99 \\
\hline DLM pós-ICP, mm & $2,75(2,43 ; 3,15)$ & $2,76(2,46 ; 3,11)$ & $2,72(2,41 ; 3,16)$ & 0,75 \\
\hline DS pós-ICP, \% & $12(10 ; 15)$ & $11(10 ; 14)$ & $12(10 ; 15)$ & 0,61 \\
\hline Stent por lesão & $1,07 \pm 0,31$ & $1,06 \pm 0,27$ & $1,08 \pm 0,49$ & 0,94 \\
\hline
\end{tabular}

$\mathrm{ACC} / \mathrm{AHA}=$ American College of Cardiology/American Heart Association; DLM = diâmetro luminal mínimo; DR = diâmetro de referência; DS = diâmetro da estenose; ICP = intervenção coronária percutânea; SCACSST = síndrome coronária aguda com supradesnível do segmento ST; SCASSST = síndrome coronária aguda sem supradesnível do segmento ST.

dos por apresentar sintomas ou, no caso de pacientes assintomáticos, por haver evidência de circulação colateral para o vaso culpado, com preservação da função ventricular e anatomia coronária favorável à recanalização.
O sucesso angiográfico foi de $99 \%$, com apenas dois insucessos técnicos (um em cada grupo) que evoluíram bem, sem intercorrências. Em ambos os insucessos as artérias abordadas estavam ocluídas. 
Sant'Anna FM, et al. Desfechos Hospitalares em Pacientes Submetidos a Intervenção Coronária Percutânea na Vigência de Síndromes Coronárias Agudas Atendidos em Unidades de Pronto Atendimento (UPAs) - Resultados de um Centro de Cardiologia Terciário. Rev Bras Cardiol Invasiva. 2010;18(1):30-6.

\section{Evolução hospitalar}

A Tabela 3 mostra a evolução hospitalar. Houve 4 óbitos hospitalares, todos no grupo síndrome coronária aguda com supradesnível do segmento ST $(4,6 \%$ vs. $0 ; P=0,17)$. Um paciente apresentou obstrução intestinal seguida de abdômen agudo e choque séptico (hérnia inguinal estrangulada); dois pacientes evoluíram com acidente vascular cerebral (um deles complicado por pneumonia e sepsis) e o último foi de causa cardíaca (infarto agudo do miocárdio com choque devido a no-reflow pós-angioplastia).

Houve ainda um caso de ataque isquêmico transitório no grupo síndrome coronária aguda com supradesnível do segmento ST e um infarto agudo do miocárdio por trombose aguda do stent no grupo síndrome coronária aguda sem supradesnível do segmento ST que necessitou de nova angioplastia. Ambos os casos evoluíram bem e tiveram alta sem maiores problemas.

\section{DISCUSSÃO}

Os resultados desse estudo confirmam que o tratamento de pacientes com síndrome coronária aguda atendidos inicialmente nas UPAs por angioplastia com implante de stent é eficaz e cursa com baixos índices de eventos cardíacos adversos maiores hospitalares. Pode-se dizer ainda que não houve diferença significativa na evolução hospitalar dos dois grupos de pacientes tratados, com ou sem supradesnível do segmento ST, ainda que isso talvez se deva ao pequeno tamanho da amostra.

Dados brasileiros recentes obtidos no Departamento de Informática do Sistema Único de Saúde (DATASUS, 2004) ${ }^{8}$ demonstram que as doenças cardiovasculares e cerebrovasculares respondem por $28 \%$ da mortalidade atual no Brasil, um fenômeno que vem ocorrendo nos últimos anos nos países em desenvol- vimento. $^{8-10}$ Dados de pesquisa revelam que nos primeiros 30 dias os índices de mortalidade dos pacientes com infarto agudo do miocárdio se comportam da seguinte forma: $50 \%$ dos pacientes morrem antes de chegar ao hospital e 25\%, nas primeiras 48 horas após a admissão hospitalar. ${ }^{9,10}$ Fica bem clara a necessidade de um tratamento adequado iniciado o mais rapidamente possível após o início dos sintomas, ainda em casa, no transporte ou mesmo em ambiente pré-hospitalar. Nesse aspecto, o Projeto UPA mostrouse de grande importância, na medida em que muitos pacientes eram buscados em suas residências pelas ambulâncias do Corpo de Bombeiros do Rio de Janeiro e o tratamento já era iniciado no próprio local. Após a chegada na UPA, o paciente é imediatamente triado por médicos e enfermeiros e atendido prontamente quando se trata de caso emergencial. Por esse motivo as UPAs acabaram se tornando os locais de referência para onde vão os pacientes com dor torácica na cidade do Rio de Janeiro. No entanto, diante da carência de leitos hospitalares pelo SUS no Estado, depois desse primeiro atendimento o paciente muitas vezes não tinha para onde ir, não podendo ser dada continuidade a seu tratamento nem tampouco se realizar estratificação de risco adequada no paciente com síndrome coronária aguda. Por isso, a parceria firmada com um hospital prestador de serviço pelo SUS no Estado veio preencher uma lacuna no tratamento de parte desses pacientes.

São inúmeros os estudos que investigaram a incidência de eventos cardíacos adversos maiores no infarto agudo do miocárdio, tanto em pacientes submetidos a trombólise química ${ }^{11,12}$ como em pacientes tratados por angioplastia primária. ${ }^{13,14}$ Nosso estudo não se encaixa em nenhum dos dois perfis acima, pois: 1) não realizamos angioplastia primária em nenhum desses pacientes; 2) os pacientes chegavam a nosso serviço relativamente estáveis, em média quatro dias após o

TABELA 3

Evolução hospitalar

\begin{tabular}{|c|c|c|c|c|}
\hline & \multirow[b]{2}{*}{$\begin{array}{c}\text { Pacientes } \\
(n=164)\end{array}$} & \multicolumn{2}{|c|}{ Grupos } & \multirow[b]{2}{*}{ Valor de $\mathbf{P}$} \\
\hline & & $\begin{array}{c}\text { SCACSST } \\
(\mathrm{n}=86)\end{array}$ & $\begin{array}{c}\text { SCASSST } \\
(n=78)\end{array}$ & \\
\hline \multicolumn{5}{|l|}{ ECAM hospitalares, n (\%) } \\
\hline Total de eventos & $6(3,6)$ & $5(5,8)$ & $1(1,3)$ & 0,60 \\
\hline Óbito & $4(2,4)$ & $4(4,6)$ & 0 & 0,17 \\
\hline IAM & $2(1,2)$ & $1(1,2)$ & $1(1,3)$ & 0,94 \\
\hline AVC & $3(1,8)$ & $3(3,5)$ & 0 & 0,25 \\
\hline RLA & $1(0,6)$ & 0 & $1(1,3)$ & 0,29 \\
\hline Trombose aguda do stent & $1(0,6)$ & 0 & $1(1,3)$ & 0,29 \\
\hline
\end{tabular}


Sant'Anna FM, et al. Desfechos Hospitalares em Pacientes Submetidos a Intervenção Coronária Percutânea na Vigência de Síndromes Coronárias Agudas Atendidos em Unidades de Pronto Atendimento (UPAs) - Resultados de um Centro de Cardiologia Terciário. Rev Bras Cardiol Invasiva. 2010;18(1):30-6.

evento agudo; e 3) havia uma mistura de pacientes, tanto com síndrome coronária aguda com supradesnível do segmento ST como com síndrome coronária aguda sem supradesnível do segmento ST. No entanto, podemos dizer que o perfil clínico de nossos pacientes era grave (34\% de diabéticos, $67 \%$ de multiarteriais), semelhante ou mesmo pior que aqueles envolvidos nos estudos randomizados, e que uma taxa de mortalidade hospitalar de 4,6\% com incidência de eventos cardíacos adversos maiores de apenas 5,8\% foi bastante satisfatória, sendo até mesmo inferior à encontrada no estudo GRACE. ${ }^{3}$

Diversos ensaios clínicos já compararam a evolução de pacientes com infarto agudo do miocárdio com e sem supradesnível do segmento ST. ${ }^{15,16}$ A maior parte dos estudos mostra que a síndrome coronária aguda com supradesnível do segmento ST carreia riscos maiores e cursa com taxas de mortalidade, reinfarto, acidente vascular cerebral e insuficiência cardíaca congestiva superiores às da síndrome coronária aguda sem supradesnível do segmento ST, conforme nos mostram os resultados do registro Organization to Assess Strategies for Ischemic Syndromes (OASIS). ${ }^{17}$ Em nosso registro os índices de eventos cardíacos adversos maiores não foram estatisticamente diferentes entre os dois grupos $(5,8 \%$ vs. $1,3 \%$; $P=0,60)$, provavelmente em decorrência do tamanho reduzido da amostra. Chamamos ainda a atenção para o fato de o infarto agudo do miocárdio sem supradesnível do segmento ST apresentar taxas de mortalidade e de sobrevida livre de eventos cardíacos adversos maiores semelhantes às do infarto agudo do miocárdio com supradesnível do segmento ST aos 12 meses de evolução, ${ }^{15,16}$ o que reforça a necessidade de estratificação precisa, de acompanhamento de perto e de tratamento intensivo dos pacientes com síndrome coronária aguda sem supradesnível do segmento ST após a alta hospitalar.

Poder-se-ia questionar o tratamento percutâneo tardio desses pacientes, que foi baseado principalmente na presença de lesão angiograficamente significativa (> 70\%) na artéria culpada, sem antes a realização de testes provocadores de isquemia. No entanto, as Diretrizes da Sociedade Brasileira de Cardiologia para Intervenção Coronária $^{6}$ consideram a angioplastia de lesões $>50 \%$ e $<100 \%$ após fibrinólise como recomendação classe IIA, nível de evidência A. Sabe-se ainda da grande dificuldade de estratificação não-invasiva e invasiva ambulatorial em pacientes do SUS, que, por vezes, pode demorar meses. ${ }^{18}$ Além disso, em estudo publicado por Sant'Anna et al., ${ }^{19}$ em 2008, os autores, utilizando o fluxo fracionado de reserva do miocárdio para definir a acurácia da angiografia em predizer se uma lesão seria ou não isquêmica, demonstraram que lesões maiores que $70 \%$ pela angiografia coronária quantitativa eram responsáveis por isquemia em 96\% dos casos, enquanto nas lesões moderadas (entre $40 \%$ e $70 \%$ ) apenas $57 \%$ foram responsáveis por isquemia miocárdica.
Como o grau de estenose médio em nosso estudo foi de $76 \%$ (pela angiografia coronária quantitativa), pareceu-nos razoável supor que as lesões abordadas eram potencialmente causadoras de isquemia e que se fossem deixadas sem tratamento adequado o índice de eventos cardíacos adversos tardios nesses pacientes poderia ser mais elevado. ${ }^{20,21}$ No caso da síndrome coronária aguda com supradesnível do segmento ST, em particular, uma meta-análise publicada em 2006 demonstrou que a realização de angioplastia após fibrinólise em pacientes com infarto agudo do miocárdio durante a internação hospitalar foi capaz de reduzir as elevadas taxas de morte ou reinfarto nesse grupo de pacientes. ${ }^{22}$

Existem algumas limitações no presente estudo. Em primeiro lugar, o tamanho reduzido da amostra para poder avaliar diferenças entre os grupos; entretanto, esse não era o objetivo principal do estudo, que era tão somente mostrar a experiência inicial com o tratamento percutâneo tardio de pacientes com infarto agudo do miocárdio atendidos nas UPAs. Em segundo lugar, não recebemos pacientes com choque cardiogênico, o que, com certeza, aumentaria nossa mortalidade geral; entretanto, pela distância e pelos meios de transporte disponíveis, esses pacientes eram encaminhados prioritariamente a hospitais públicos no próprio município do Rio de Janeiro, pois trazê-los para nosso hospital acarretaria grande risco, já que esses pacientes estavam hemodinamicamente muito instáveis.

\section{CONCLUSÃO}

Este estudo demonstrou que o tratamento percutâneo tardio dos pacientes com infarto agudo do miocárdio atendidos nas UPAs cursa com excelentes resultados hospitalares e baixos índices de complicação. É necessário agora o seguimento posterior desses pacientes para verificar se esse benefício se mantém ao longo do tempo.

\section{CONFLITO DE INTERESSES}

Os autores declararam inexistência de conflito de interesses relacionado a este artigo.

\section{REFERÊNCIAS}

1. Murray CJL, Lopez AD. The global burden of disease: a comprehensive assessment of mortality and disability from disease, injuries and risk factors in 1990 and projected to 2020. Cambridge: Harvard University Press; 1996.

2. Lopez AD. Assessing the burden of mortality from cardiovascular diseases. World Health Stat Q. 1993;46:91-6.

3. Steg PG, Goldberg RJ, Gore JM, Fox KA, Eagle KA, Flather MD, et al.; GRACE Investigators. Baseline characteristics, management practices, and in-hospital outcomes of patients hospitalized with acute coronary syndromes in the Global Registry of Acute Coronary Events (GRACE). Am J Cardiol. 2002;90(4):358-63.

4. Myocardial infarction redefined - a consensus document of the Joint European Society of Cardiology/American College of Cardiology Committee for the redefinition of myocardial infarction. Eur Heart J. 2000;21(18):1502-13. 
Sant'Anna FM, et al. Desfechos Hospitalares em Pacientes Submetidos a Intervenção Coronária Percutânea na Vigência de Síndromes Coronárias Agudas Atendidos em Unidades de Pronto Atendimento (UPAs) - Resultados de um Centro de Cardiologia Terciário. Rev Bras Cardiol Invasiva. 2010;18(1):30-6.

5. Piegas LS, Timerman A, Feitosa G, Mattos LA, Nicolau JC, Rossi Neto JM, et al. Sociedade Brasileira de Cardiologia. Diretriz da Sociedade Brasileira de Cardiologia sobre Tratamento do Infarto Agudo do Miocárdio com Supradesnível do Segmento ST. Arq Bras Cardiol. 2009;93(6 Supl 2):e179-e264.

6. Mattos LA, Lemos Neto PA, Rassi A Jr, Marin-Neto JA, Sousa AGMR, Devito FS, et al. Diretrizes da Sociedade Brasileira de Cardiologia - Intervenção Coronária Percutânea e Métodos Adjuntos Diagnósticos em Cardiologia Intervencionista (II Edição - 2008). Arq Bras Cardiol. 2008;91(6 Supl 1):1-58.

7. Reiber $\mathrm{JH}$, Serruys PW, Kooijman CJ, Wijns W, Slager CJ, Gerbrands JJ, et al. Assessment of short-, medium-, and longterm variations in arterial dimensions from computer-assisted quantitation of coronary cineangiograms. Circulation. 1985; 71(2):280-8.

8. DATASUS. Ministério da Saúde. Sistema de Informações sobre Mortalidade - SIM. Estatísticas Vitais - Mortalidade e Nascidos Vivos. Disponível em: http://www.datasus.gov.br.

9. Avezum A, Neto JMR, Piegas L. Why do we need randomized and epidemiological studies on cardiovascular disease? Evidencebased cardiology VII. Arq Bras Cardiol. 1999;72(3):289-95.

10. Mansur AP, Souza MFM, Favarato D, Avakian SD, Nicolau JS, César LAM, et al. Tendência da mortalidade por doenças cardiovasculares no Brasil de 1979 a 1996. Arq Bras Cardiol. $2001 ; 76: 497-503$

11. In-hospital mortality and clinical course of 20,891 patients with suspected acute myocardial infarction randomised between alteplase and streptokinase with or without heparin. The International Study Group. Lancet. 1990;336(8707):71-5.

12. van de Werf F, Adgey J, Ardissino D, Armstrong PW, Aylward $P$, Barbash G, et al. Single-bolus tenecteplase compared with front-loaded alteplase in acute myocardial infarction: the ASSENT-2 double-blind randomised trial. Assessment of the Safety and Efficacy of a New Thrombolytic Investigators. Lancet. 1999;354(9180):716-22.

13. Grines CL, Cox DA, Stone GW, Garcia E, Mattos LA, Giambartolomei A, et al. Coronary angioplasty with or without stent implantation for acute myocardial infarction. Stent Primary Angioplasty in Myocardial Infarction Study Group. N Engl I Med. 1999;341:1949-56.
14. Stones GW, Grines CL, Cox DA, Garcia E, Tcheng JE, Griffin $\mathrm{J}$, et al. Comparison of angioplasty with stenting, with and without abciximab, in acute myocardial infarction. The Controlled Abciximab and Device Investigation to Lower Late Angioplasty Complications (CADILLAC) Investigators. N Engl J Med. 2002;346:957-66.

15. Sim DS, Kim JH, Jeong MH. Differences in clinical outcomes between patients with ST-elevation versus non-ST-elevation acute myocardial infarction in Korea. Korean Circ J. 2009; 39(8):297-303

16. Manari A, Albiero R, De Servi S. High-risk non-ST-segment elevation myocardial infarction versus ST-segment elevation myocardial infarction: same behaviour and outcome? J Cardiovasc Med (Hagerstown). 2009;10 Suppl 1:S13-6.

17. Piegas LS. Infarto agudo do miocárdio não-Q e angina instável: estudo comparativo entre diferenças clínicas e regionais [tese de livre-docência]. São Paulo: Faculdade de Medicina da Universidade de São Paulo; 1999.

18. Gontijo RV, Rezende NA. Comparação da adequação das indicações de cinecoronariografias diagnósticas eletivas entre os sistemas de saúde público e privado no Estado de Minas Gerais. Epidemiol Serv Saúde. 2008;17(2):128-30.

19. Sant'Anna FM, Silva ER, Batista LA, Brito MB, Ventura FM, Ferraz HA, et al. Qual o erro da angiografia na definição de isquemia miocárdica durante intervenções coronarianas percutâneas? Arq Bras Cardiol. 2008;91(3):179-84.

20. Pijls NH, van Schaardenburgh P, Manoharan G, Boersma E, Bech JW, van't Veer M, et al. Percutaneous coronary intervention of functionally nonsignificant stenosis: 5-year follow-up of the DEFER Study. J Am Coll Cardiol. 2007 May $29 ; 49(21): 2105-11$.

21. Tonino PA, De Bruyne B, Pijls NH, Siebert U, Ikeno F, van't Veer M, et al.; FAME Study Investigators. Fractional flow reserve versus angiography for guiding percutaneous coronary intervention. N Engl J Med. 2009;360(3):213-24.

22. Collet JP, Montalescot G, Le May M, Borentain M, Gershlick A. Percutaneous coronary intervention after fibrinolysis: a multiple meta-analyses approach according to the type of strategy. J Am Coll Cardiol. 2006;48(7):1326-35. 